\title{
Management of Partially Accommodative Esotropia: A case report
}

\section{Rani Pitta $^{1 *}$, Mayasari Wahyu ${ }^{1,2}$, Feti Karfiati ${ }^{1,2}$, Irawati Irfani ${ }^{1,2}$, Primawita Amiruddin ${ }^{1,2}$, Sesy Caesarya ${ }^{1,2}$}

${ }^{1}$ Department of Ophthalmology, Faculty of Medicine, Universitas Padjadjaran, Indonesia National Eye Centre Cicendo Eye Hospital

${ }^{2}$ Cicendo Eye Hospital National Eye Center, Bandung, Indonesia.

*Corresponding Author: Rani Pitta, Department of Ophthalmology, Faculty of Medicine, Universitas Padjadjaran, Indonesia National Eye Centre Cicendo Eye Hospital, Indonesia.

Received date: 26 August 2021; Accepted date: 06 September 2021; Published date: 10 September 2021

Citation: Pitta R, Wahyu M, Karfiati F, Irfani I, Amiruddin P, et al. (2021) Management of Partially Accommodative Esotropia: A case report. J Med Case Rep Case Series 2(10): https://doi.org/10.38207/JMCRCS/2021/0208140

Copyright: (C) 2021 Rani Pitta. This is an open-access article distributed under the terms of the Creative Commons Attribution License, which permits unrestricted use, distribution, and reproduction in any medium, provided the original author and source are credited.

\begin{abstract}
Background

Partially accommodative esotropia show a reduction in the angle of esotropia when wearing spectacles but have a residual esotropia despite the provision of the full hyperopic correction. Amblyopia is common and thus the chance for achieving fusion and high-grade stereopsis is usually not high in this group. This case to report management of partially accommodative esotropia with amblyopia in children.

\section{Case presentation}

Six years old girl came with her mother to the Pediatric and Strabismus division of Cicendo

National Eye Hospital with a chief complaint of esodeviation in the left eye 4 years ago. No previous history of trauma or illness.

Lensometer result RE S +1.50 C-1.25 x140 LE S+4.25 C-3.00 x170. The best-corrected visual acuity was 0.63 on RE and 0.5 on LE. Ocular movements were good in all directions. Hirschberg test without spectacles showed left esotropia 300 and with spectacles left esotropia 150. Worth Four Dot Test (WFDT) and TNO test showed suppression in LE with stereopsis more than 2000 minutes of arc, respectively anterior and posterior segment within the normal limit in both eyes. She was diagnosed with partially accommodative esotropia. She underwent bilateral medial rectus recession to treat partially accommodative esotropia. There was an improvement in the patient's eye alignment. She had orthotropic eye alignment with spectacle correction of the full amount of hyperopia after one week follows up.

\section{Conclusions}

The goal therapy for partially accommodative esotropia is not to operate the patient out of spectacles, but rather to achieve alignment and fusion with full hyperopia correction.
\end{abstract}

Keywords: accommodative esotropia, strabismus, amblyopia.

\section{Introduction}

Esodeviation is a latent or persistent convergent visual axis misalignment. Esodeviation is the most common form of strabismus in children, around $50 \%$ of the ocular deviation in the pediatric population. Esotropia is divided into accommodative and nonaccommodative. The accommodative esotropia is a convergent deviation of the eye associated with activation of the accommodation reflex. Accommodative esotropia is divided into refractive and nonrefractive. The accommodative refractive esotropia is divided into two parts, namely fully and partially.[1-3]

Partially accommodative esotropia is a form of accommodative esotropia characterized by the presence of residual esodeviations after full correction of hypermetropia. This condition can be diagnosed in children aged 18-48 months. The causes of partially accommodative esotropia are different degrees of hypermetropia, anatomical factors, congenital fusion dysfunction, and contracture of the medial rectus muscle.[1,3-4]

The uncorrected hypermetropic refractive abnormality can cause excessive accommodation to focus the image on the retina so that the convergence power is not proportional to the divergence force and ultimately leads to an esotropic state. Therapy of partially accommodative esotropia is the full correction of hypermetropia and strabismus surgery for the management of residual esodeviation.

[2-5] This case report will discuss the management of partially accommodative esotropia. 


\section{Case Report}

A 6-year-old girl came to the Pediatric Ophthalmology and Strabismus unit on December 12, 2019, with a complaint of inward crossed left eye from the age of 2.5 years. This condition persists even after wearing spectacles. The condition of the squint was initially small but has been increasing for the last 2 years. Patients tend to tilt their heads to the left when they want to focus on seeing something from a distance, her parents didn't remember the size of the spectacles they were first wearing. The patient has been wearing spectacles since 2 years ago. The spectacles have been used for the last 1 year and are still comfortable for patients. The patient has been routinely treated for the occlusion of the right eye 4 hours a day for 1 year.

History of trauma to the eye, previous eye surgery, and previous systemic disease was denied by the family. There is no family history of strabismus. No previous double vision complaints. The patient is the second child of 2 siblings. Full-term birth, normal delivery by an obstetrician, birth weight 3300 grams. Complete immunization history with the pediatrician. Normal history of child development. Antenatal care was routinely done at the doctor. During the pregnancy, the patient's mother denied a history of illness and takes certain drugs other than the doctor. The patient had a physical examination within normal limits generally. Uncorrected visual acuity of the right eye was 0.2 and 0.05 for the left eye with the Snellen chart. The best-corrected visual acuities with her spectacles were 0.63 for the right eye and 0.5 for the left eye. The results of the lensometer on the right eye were $\mathrm{S}+1.50 \mathrm{C}-1.25$ x140 and the left eye was $\mathrm{S}+4.25 \mathrm{C}-3.00 \times 170$. The cyclorefraction examination showed that the right eye was $S+2.00 \mathrm{C}-1.00 \times 150=0.63$ and the left eye was $\mathrm{S}+5.00 \mathrm{C}-3.50 \times 170=0.5$.

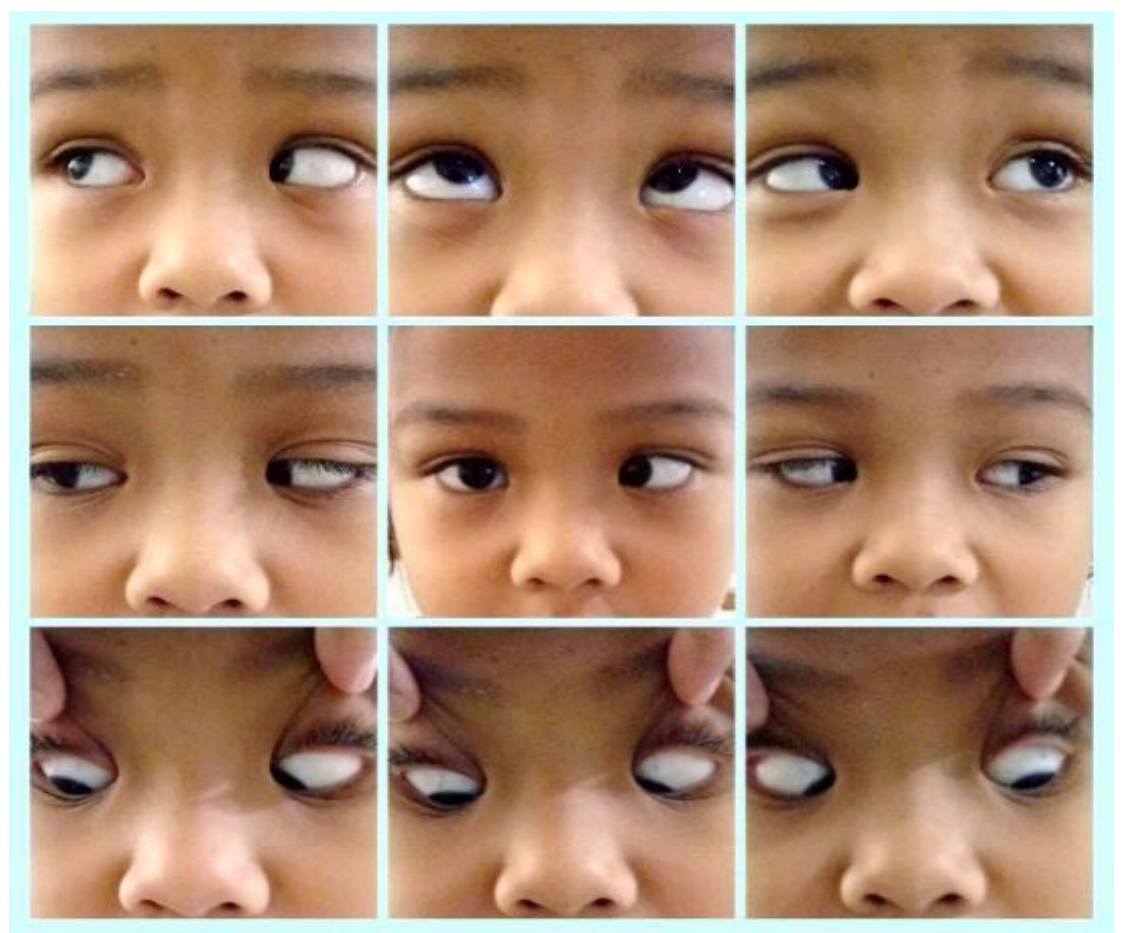

Figure 2.1 Picture of 9 gaze positions pre surgery

Version and duction movement of the eyeballs were good in all directions except the movement of the left eye towards the nasal +1 . The position of the left eyeball without spectacles was esotropia $30^{\circ}$ and esotropia $15^{\circ}$ with spectacles. The cover/ uncover alternate cover test examinations revealed esotropia alternans with a dominant fixation on the right eye. There was no nystagmus. Near sensory function examination with TNO (Toegepast Natuurwetenschappelijk Onderzoek) test and distance sensory function with Worth 4-dot testing (WFDT) obtained suppression in the left eye. Stereopsis examination with TNO test> 2000 second of arc.

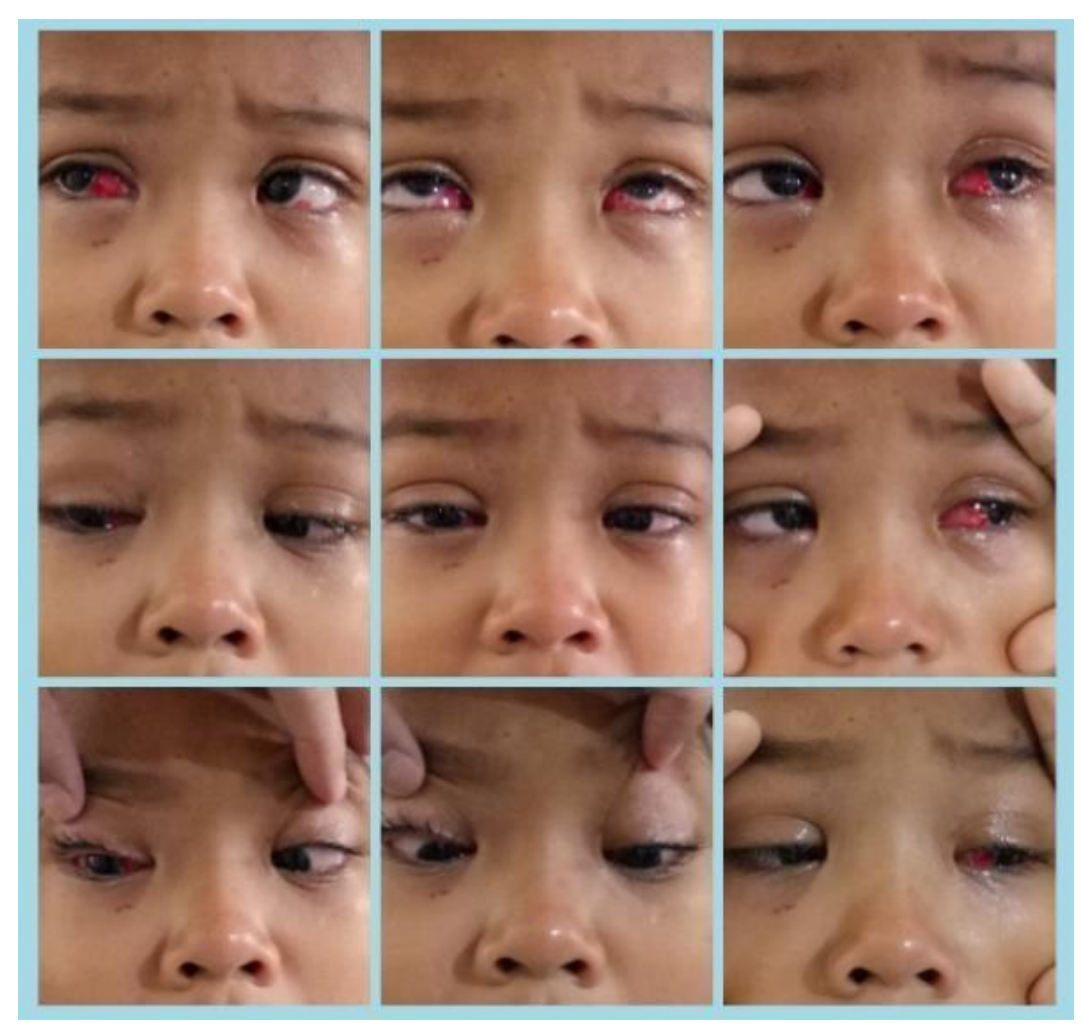

Figure 2.2 Picture of 9 gaze positions first day after surgery 


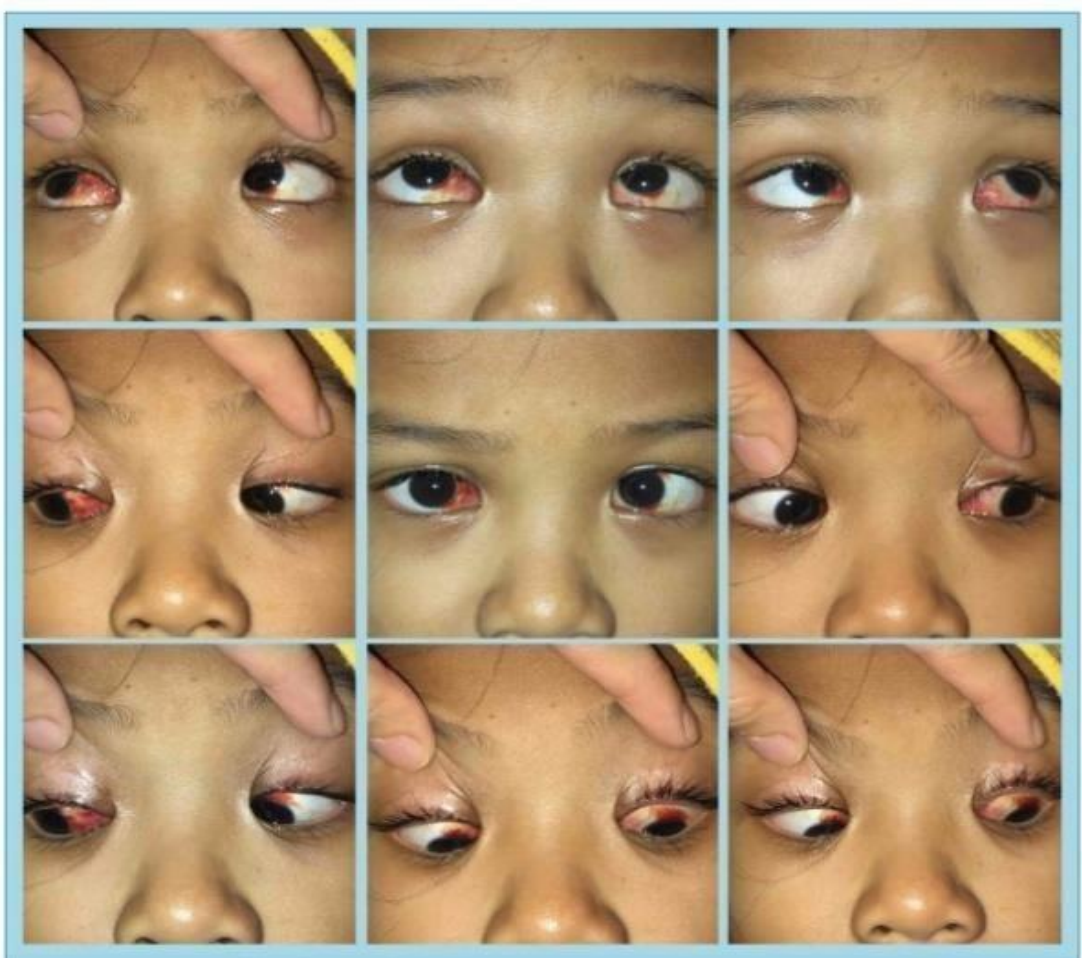

Figure 2.3 Picture of 9 gaze positions one week after surgery

The result of prism and alternate cover test (PACT) at 3 positions (primary, upward, and downward position) without spectacles were obtained for near $50 \Delta$ base out (BO) and distances $45 \Delta$ BO. PACT with spectacles results in near distance $45 \Delta \mathrm{BO}$ and distance $40 \Delta \mathrm{BO}$. The anterior and posterior segments of both eyes were within normal limits. Patients were diagnosed with partially accommodative esotropia, amblyopia strabismus, and compound hyperopic astigmatism.

The patient underwent bilateral medial rectus muscle recession in general anesthesia on 17 December 2019. The medial rectus muscle was recessed $6 \mathrm{~mm}$ in each eye. The results obtained on the first day after surgery were the position of the eyeballs without esotropia spectacles was $15^{\circ}$ and with esotropia spectacles were $7^{\circ}$. On examination of the anterior segment, subconjunctival hemorrhage and intact sutures were found in the conjunctiva in both eyes. Patients were asked to return 1 week after surgery to follow up.

The follow up 1 week later showed that uncorrected visual acuity of the right eye was 0.2 and the left eye was 0.05 using the Snellen chart. Best-corrected visual acuities used old spectacles were
0.63 for the right eye and 0.5 for the left eye. Version and duction movement of both eyeballs were good in all directions. The eyeballs position without spectacles correction was esotropia and $7^{\circ}$ with orthotropic spectacles. The result of the PACT examination with spectacles obtained results both $4 \Delta \mathrm{BO}$ in near and distance. The anterior segment revealed subconjunctival hemorrhage and intact sutures in the conjunctiva of both eyes. The patient was asked to return in 2 weeks.

The patient returned to follow up 4 weeks after the surgery. The results of cyclorefraction correction showed; OD: $\mathrm{S}+2.75 \mathrm{C}-1.50$ x160: 0.63 and OS:S+5.00 C-3.00 x170: 0.5. The position of the eyeballs with spectacles resulted in orthotopic. The result of the cover / uncover test was esophoria. PACT result with the use of near vision deviation spectacles was $10 \Delta \mathrm{BO}$. The patient was given newly corrected spectacles based on cyclorefraction correction and occlusion therapy in the right eye was continued 4 hours a day. Patients were asked to return within 1 month.

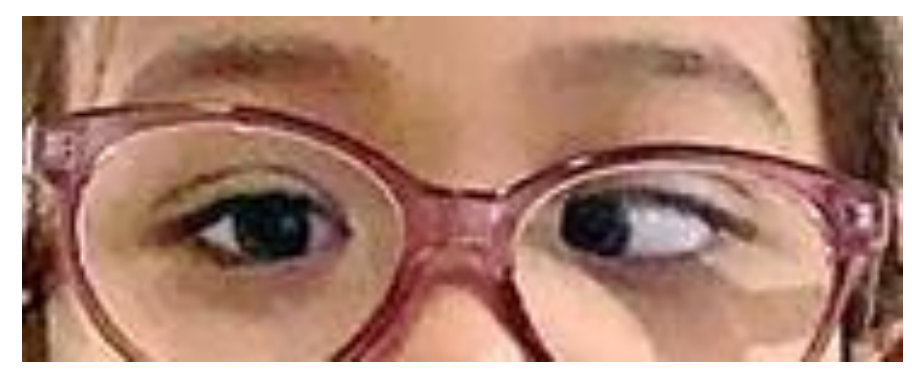

(A) (B)

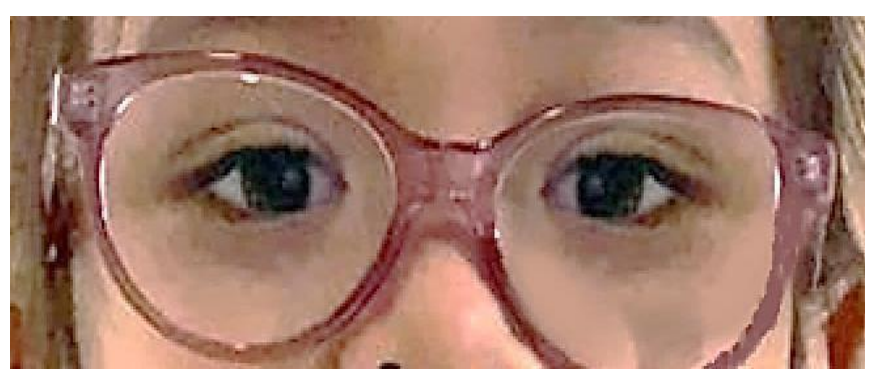

Figure 2.4 Position of eyeballs with spectacles

(A) pre-surgery

(B) one month after surgery 
Esodeviation is the most common form of strabismus in children, around $50 \%$ of the ocular deviation in the pediatric population. This condition can be diagnosed in children aged 18-48 months. [3,5-7] Based on a history of the patient's mother, complaint of inwardsquinting left eye began to appear at the age of 2.5 years old.

Accommodative esotropia are divided into refractive and nonrefractive. Patients with uncorrected (refractive) hypermetropia must accommodate to get clear images. This condition will stimulate convergencebut is not matched by the amplitude of the divergence. The accommodative refractive esotropia is subdivided into two parts, fully and partially. The fully accommodative refractive type is characterized by the loss of esodeviation after wearing spectacles while the partially accommodative refractive type shows residual modelization after wearing corrected spectacles. $[\mathbf{2 , 4 , 6 - 8}]$ In this case, after wearing corrected spectacles from the age of 2.5 years accompanied by occlusion therapy for 1 year, there is still a residual deviation found

Harley et al. stated that the partially accommodative condition of esotropia is not purely due to accommodative factors such as differences in the degree of hypermetropia (refractive). Nonaccommodative conditions such as anatomical factors, congenital fusion dysfunction, and contracture of the medial rectus muscle can be the partially accommodative causes of esotropia. Refractive abnormalities followed by a normal AC/A (accommodative convergence to accommodation) ratio. The degree of hypermetropia is usually above $+4.00 \mathrm{D}$ with a deviation of $20-30 \Delta$ and does not differ in far and near vision. Nathalie et al. stated that the deviation in partially accommodative refractive esotropia varies but is usually greater than fully accommodative esotropia. The deviation of near and far-distance vision usually does not differ. [4,6- 7,9] In this patient, a high hypermetropic refractive condition in the left eye and a residual deviation with correction were found. PACT with spectacles obtained residual with results $45 \Delta \mathrm{BO}$ and $40 \Delta \mathrm{BO}$ for near and distance respectively.

Development of stereopsis function begins at the age of 3 months and undergoes significant maturation between the ages of 8-18 months and continues to develop until the age of 36 months. Seventy-five percent of patients with partially accommodative esotropia have binocular vision problems even though this condition occurs above that critical period. Sensory function tests examination using the

Table 1: Numbers of Esodeviation Surgery
Worth 4-dot testing (WFDT) and stereopsis. WFDT is an examination to assess the presence of suppression in one eye while stereopsis function is using the TNO or Stereo Fly test. Lee et al stated that stereoptic vision can still be obtained if near vision deviation is $\leq 4 \Delta$ and distance vision $\leq$ is $5 \Delta$. [2,7,9-11]. In our case, the patient had fusion disorders on a near examination performed with TNO. Stereopsis examination performed using the TNO test showed $>2000$ seconds of arc, so it can be said that the binocular sensory function in this patient is impaired. Residual deviation with spectacles in the patient has obtained $45 \Delta \mathrm{BO}$ for near and $40 \mathrm{BO}$ for distance.

Esodeviative management aims to restore ocular alignment, improve visual acuity, and improve binocular function. The initial management of partially accommodative esotropia is to correct the patient's abnormality based on the maximum correction of the cycloplegic condition. Abdi et al stated that maximum hypermetropic correction can be done by giving spectacles or contact lenses. The irregular wearing of spectacles can lead to poor motor and sensory function improvement. Treatment of amblyopia begins with providing optical correction according to goodcycloplegic refraction, followed by classic amblyopia therapy, by occluding or closing the dominant eye as early as possible, to achieve the maximum result. Wilson et al explained that besides getting the alignment expected, partially accommodative esotropia patients had improved stereoptic vision. $[1,3,5,12]$ In this case, the patient had been wearing spectacles since the age of 2.5 years and had routinely performed occlusion therapy for the last 1 year but there was still a deviation.

Kenneth et al stated that a residual deviation of more than $10 \Delta$ with correction for maximal hypermetropia is an indication for strabismus surgery in patients with partially accommodative esotropia. The goal of surgery is not to make the patient spectacles-free but to obtain ocular alignment and improve binocular vision function by wearing fully corrected spectacles. Bilateral medial rectus muscle recession is the procedure of choice for partially accommodative esotropia. The patient had a $6 \mathrm{~mm}$ bilateral recession of the medial rectus muscle in each eye. Kenneth et al stated that the amount of muscle that was recessed could be calculated based on the augmentation formula, the sum of deviation of the near without spectacles, and distance deviation with spectacles, divided by 2.[3,9-11,13]. In our patient, the deviation found to be $45 \Delta(50 \Delta+40 \Delta \mathrm{BO} / 2)$ then the recession is carried out at about $6 \mathrm{~mm}$ as shown in the table (Table 1).

\begin{tabular}{|l|l|l|l|}
\hline Angle of Esotropia & Recession MR OU, mm & or & Resection LR OU mm \\
\hline 15 & 3.0 & 4.0 \\
\hline 20 & 3.5 & & 5.0 \\
\hline 25 & 4.0 & 6.0 \\
\hline 30 & 4.5 & 7.0 \\
\hline 35 & 5.0 & 8.0 \\
\hline 40 & 5.5 & 9.0 \\
\hline 50 & 6.0 & & 9.0 \\
\hline
\end{tabular}

*LR = lateral rectus, $\mathrm{MR}=$ medial rectus, $\mathrm{OU}=$ both eyes (oculi uterque) Source: Cantor $^{1}$ 
In et al. stated that the expected result of surgery is the residual deviation of near and distance vision $\leq 10 \Delta$. Scott et al. stated that the risk factors for failure after surgery on partially accommodative esotropia were large deviations, high hyperopia, age too young, and the presence of amblyopia. Li et al. stated that spectacles correction $\leq$ 6 months after the onset of esotropia was one of the success factors of surgery compared to spectacles correction $>6$ months after the onset of esotropia.[2,7-9,13] The follow-up at week 3 obtained a good eye position with the cover/ uncover test esophoria.PACT result with the use of old correction spectacles obtained near vision deviation of $10 \Delta$ BO.

\section{Conclusion}

Full correction of hypermetropia as well as the dominant eye occlusion is the main therapy of partially accommodative esotropia. Strabismus surgery aims to correct any residual deviation from wearing spectacles in order to obtain ocular alignment and improve binocular vision function. Adherence to the use of spectacles and amblyopia therapy after surgery is needed to support the success of

\section{References}

1. Cantor B, Christopher J, George A. Esodeviations. Dalam : American academy of ophthalmology pediatric ophthalmology and strabismus. Bab 8. San Fransisco. 2017-2018. p56-60

2. Li B, Sapna Sharan (2019) Post-operative analysis of pediatric esotropia associated with high hypermetropia. BMC Ophthalmology. 19(1): 140.

3. Wright KW, Strube YNJ (2015) Color atlas of strabismus surgery strategies and techniques. p23-8

4. Hoyt, C., Taylor, D., Lambert, S. Accommodative esotropia. USA: Elsevier, 2017. p802-5

5. Abdi S, Thunholm-Henriksson I-L, Pansell T (2016) Stepwise increase of hypermetropic correction using contact lenses in intermittent partially accommodative esotropia: Contact lenses in accommodative esotropia. 99(3): 258-63.

6. Narula A, Singh S (2018) Esotropia. J Ophthalmol. 3(4): 1-5.

7. Nelson LB, Olitsky SE (2014) Harley's pediatric ophthalmology. Philadelphia. p140-50.

8. Oh SY, Lee J-Y, Park K-A, Oh SY (2016) Long- term changes in refractive error and clinical evaluation in partially accommodative esotropia after surgery. PLoS ONE. 11(12):
In this case, the patient still needs long-term follow-up to see the success of surgical therapy, full correction spectacles, and occlusion therapy. The patient's parents were educated so that the child continues to wear spectacles to maintain ocular alignment and occlusion therapy for the management of amblyopia. Hye et al. suggested a minimum follow-up of 1 year to determine whether the patient's hypermetropia correction can maintain eye alignment after surgery. Prognosis of this the patient is still good even in the presence of strabismus amblyopia which is characterized by thesuppression of the left eye and the full correction spectacles showed no optimal visual acuity improvement. [8-9,12]

therapy so that it can achieve better visual acuity, alignment of the eyeballs, and binocularity.

Declarations: There are no financial conflicts of interest to disclose.

$\mathrm{e} 0166695$.

9. Choe HR, Yang HK, Hwang J-M (2019) Long-term outcomes of prismatic correction in partially accommodative esotropia. PLoS ONE. 14(12): e0225654.

10. Iordanous Y, Mao A, Makar I (2015) Preoperative factors affecting stereopsis after surgical alignment of acquired partially accommodative esotropia. Strabismus. 23(4): 151-8.

11. Kurup SP, Barto HW, Myung G, Mets MB (2018) Stereoacuity outcomes following surgical correction of the nonaccommodative component in partially accommodative esotropia. Journal of american association for pediatric ophthalmology and strabismus. 22(2): 92-96.

12. Esposito Veneruso P, Bruzzese D, Magli A (2018) Long-term development of refractive error in refractive, nonrefractive and partially accommodative esotropia. PLoS ONE. 13(9): e0204396.

13. Jiang D, Han D, Zhang J, Pei T, Zhao Q (2018) Clinical study of the influence of preoperative wearing time on postoperative effects in children with partially accommodative esotropia: Medicine. 97(19): e0619. 